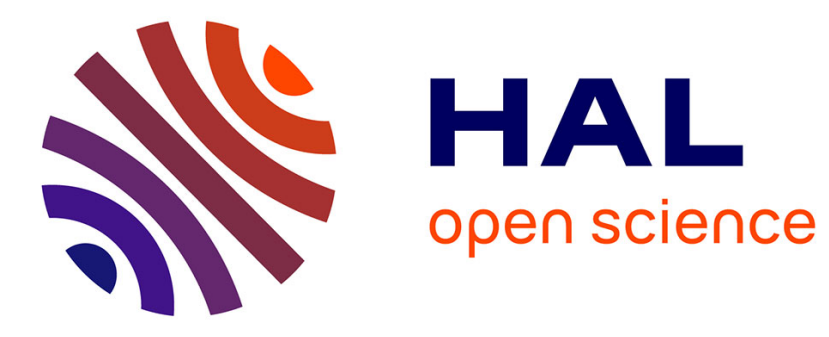

\title{
Du problème de Molyneux au problème de Bach-y-Rita
}

\author{
Elisabeth Pacherie
}

\section{To cite this version:}

Elisabeth Pacherie. Du problème de Molyneux au problème de Bach-y-Rita. Joëlle Proust. Perception et Intermodalité, Approches actuelles du Problème de Molyneux, Paris: PUF, pp.255-293, 1997, 9. ijn_00000242

\section{HAL Id: ijn_00000242 \\ https://hal.science/ijn_00000242}

Submitted on 28 Oct 2002

HAL is a multi-disciplinary open access archive for the deposit and dissemination of scientific research documents, whether they are published or not. The documents may come from teaching and research institutions in France or abroad, or from public or private research centers.
L'archive ouverte pluridisciplinaire HAL, est destinée au dépôt et à la diffusion de documents scientifiques de niveau recherche, publiés ou non, émanant des établissements d'enseignement et de recherche français ou étrangers, des laboratoires publics ou privés. 
Pacherie, E. 1997. Du problème de Molyneux au problème de Bach-y-Rita. In J. Proust (ed.), Perception et Intermodalité, Approches actuelles du Problème de Molyneux,, Paris, PUF, pp. 255-293.

\title{
DU PROBLÈME DE MOLYNEUX AU PROBLÈME DE BACH-Y-RITA 1
}

\author{
Elisabeth PACHERIE \\ pacherie@ehess.fr
}

Quelle est la nature et l'origine de nos représentations spatiales ? La perception visuelle a-t-elle un caractère immédiatement spatial ? Différentes modalités sensorielles peuvent-elles être porteuses d'une information spatiale commune? Quel est le rôle joué par la motricité dans la construction de représentations spatiales visuelles ou tactiles ? $\mathrm{Au}$ dix-huitième siècle, le débat sur ces questions s'est cristallisé autour du célèbre problème de Molyneux. La fascination qu'il a exercée sur les plus grands esprits du temps - parmi lesquels, pour ne citer que les noms les plus célèbres, Locke, Berkeley, Hume, Leibniz, Condillac, Diderot, ou encore Voltaire - tient, ainsi que l'ont expliqué Cassirer (1932) et Hatfield (1990) à ce que s'y rejoignent les interrogations alors les plus centrales de la psychologie et de la théorie de la connaissance. Comme Hatfield (1990 : 2) le souligne, la plupart des philosophes du XVIIIème siècle établissaient un lien étroit entre théorie de l'esprit et théorie de la science, l'investigation des pouvoirs ou facultés de l'esprit étant censée nous éclairer sur le contenu possible ou à tout le moins sur les limites de la connaissance en général et de la connaissance scientifique en particulier. La question de la connaissance de l'espace par l'esprit humain faisait l'objet d'un intérêt d'autant plus soutenu que les propriétés géométriques et spatiales jouaient un rôle fondamental dans la nouvelle physique galiléenne, puis newtonienne. Cette fascination

${ }^{1}$ J'ai présenté des versions antérieures de ce texte lors d'une séance du Séminaire d'Épistémologie Comparative à Aix-en-Provence, dans un atelier sur le thème "Neurosciences et philosophie: les niveaux d'intégration de I'action", organisé par J.-L. Petit au Centre d'Analyse des Savoirs Contemporains à Strasbourg et lors d'une journée sur la perception organisée par J. Proust au CREA à Paris. Ces exposés ont donné lieu à des discussions fructueuses et je remercie vivement les personnes présentes en ces occasions. Je souhaite égal ement exprimer ma gratitude envers Roberto Casati, François Clémentz, Pierre Livet, Joëlle Proust et Elisabeth Schwartz pour leurs commentaires et suggestions. 
pour le problème de Molyneux s'explique sans doute aussi par la raison complémentaire qu'il alimentait un fantasme de philosophe : qu'une interrogation philosophique puisse être tranchée de manière décisive par des moyens empiriques, en l'occurrence, nous y reviendrons, les opérations de la cataracte.

Si le problème de Molyneux continue aujourd'hui à susciter l'intérêt, le paysage intellectuel s'est toutefois beaucoup modifié. Les relations entre philosophie et psychologie ont perdu leur "innocence" pré-kantienne. Même si l'on peut refuser l'identification entre approche philosophique et approche normative, d'une part, et entre approche naturaliste et approche psychologique, de l'autre, on ne peut plus ignorer la distinction entre question de droit et question de fait. A l'intérieur de la philosophie ellemême, la conception des relations entre philosophie de l'esprit et épistémologie s'est largement transformée et l'on ne s'attend plus guère à ce que la psychologie de la perception spatiale nous éclaire sur la géométrie de l'espace physique ou l'étude de l'esprit en général sur les propriétés fondamentales du monde physique2 $\mathrm{Au}$ dixneuvième siècle, la psychologie empirique est apparue en tant que discipline autonome par rapport à la philosophie. Elle a depuis lors développé une méthodologie expérimentale riche et complexe et des variantes de la question de Molyneux reposant sur des présupposés moins problématiques que la question originale ont été conçues et ont fait l'objet d'expériences contrôlées.

La majorité des recherches expérimentales récemment menées suggèrent une réponse positive à la question de Molyneux ou plus exactement aux variantes plus facilement testables empiriquement de la question originale. Toutefois, il serait naïf d'imaginer que l'interprétation que l'on doit donner de cette réponse positive et que les conséquences qu'il faut en tirer soient parfaitement claires et dénuées d'ambiguïté. Je ne chercherai évidemment pas à démêler tout l'écheveau de ces conséquences, mais je m'intéresserai à un type particulier de variante qui a l'avantage à la fois de permettre une dissociation claire d'aspects souvent mal distingués du problème de Molyneux et de mettre en évidence l'importance du rôle de la motricité dans la construction des représentations spatiales. L'existence de prothèses sensorielles multimodales a rendu possible la formulation de certaines variantes nouvelles du problème de Molyneux. J'examinerai l'une d'entre elles, le problème de Bach-y-Rita, qu'a inspirée la mise au point par Bach-y-Rita et ses collaborateurs d'un système de substitution visuo-tactile. A partir de cette variante, je me concentrerai sur les deux problèmes suivants : (1) le problème de la relation entre sensation et perception et, corrélativement, de la base d'une classification des différents sens et (2) le problème du rôle du mouvement et de la

${ }^{2}$ Cf. Hatfield, $1990: 3$. 
motricité dans la perception spatiale (de leur contribution épistémique à la perception spatiale).

\section{La formulation classique du problème de Molyneux}

Toutefois, avant d'en arriver à ces deux questions et afin de mieux voir en quoi l'analyse de ce type de dispositif renouvelle le débat autour du problème de Molyneux, je commencerai par esquisser à grands traits l'espace de jeu philosophique dans lequel s'est inscrit au XVIIIe siècle la problème de Molyneux, en prenant pour point de référence la position défendue par Berkeley. Traditionnellement, toute discussion du problème de Molyneux commence par un rappel de la question que l'homme de loi dublinois William Molyneux soumit dans une lettre de 1688 à son ami et correspondant John Locke et que ce dernier reproduisit, en 1690, dans l'Essai sur l'entendement humain. Voici donc cette question :

Supposez un aveugle de naissance, qui soit présentement homme fait, auquel on ait appris à distinguer par le seul attouchement un cube d'un globe, du même métal et à peu près de la même grosseur, en sorte que lorsqu'il touche l'un et l'autre il puisse dire quel est le cube et quel est le globe ; supposez que le cube et le globe étant posés sur une table, cet aveugle vienne à jouir de la vue. On demande si en les voyant sans les toucher, il pourra les discerner, et dire quel est le globe et quel est le cube. (II, ix, 8).

L'irrésistible attrait de la question tient sans doute à ce qu'elle possède deux caractéristiques rarement conjointes : se situer à l'intersection des interrogations les plus centrales de la psychologie et de l'épistémologie (dans la double acception du terme) et sembler appeler une réponse empirique simple. En bref, le problème de Molyneux donne l'apparence de pouvoir constituer une expérience cruciale, comparable en son genre à l'expérience de Foucault, en ce qu'elle départagerait les théories de la connaissance et en particulier de la perception spatiale comme l'expérience de Foucault la théorie corpusculaire et la théorie ondulatoire de la lumière. Comme nous le verrons, cette apparence est trompeuse. D'une part, les opérations de la cataracte n'apportèrent pas les réponses décisives qu'on en attendait; d'autre part, les débats théoriques qui se développèrent tout au long du siècle firent ressortir la grande complexité du problème posé, montrant qu'une réponse positive comme une réponse négative à la question de Molyneux étaient susceptibles de multiples interprétations et que toute interprétation déterminée reposait sur une série d'hypothèses auxiliaires disputées par d'autres. Ce qui en fin de compte fut aussi le sort de l'expérience de Foucault. 
Je vais m'intéresser au débat théorique et essayer de dégager les principaux paramètres en jeu. La réponse négative apportée par Berkeley à la question de Molyneux et les attendus qui motivent cette réponse me serviront de points de référence. 3 Dans l'Essai pour une nouvelle théorie de la vision, le problème de Molyneux est explicitement mentionné et traité dans les sections §132-137, mais les capacités perceptives des aveugles-nés sont évoquées précédemment à plusieurs reprises et la réponse donnée par Berkeley se fonde sur des thèses déjà longuement discutées et argumentées dans les sections précédentes. Selon Berkeley, les idées immédiates de la vue et du toucher sont totalement hétérogènes. Les idées de la vue ne possèdent aucune spatialité intrinsèque. Les idées spatiales ont leur source uniquement dans le toucher et le mouvement (essentiellement l'exploration haptique et la proprioception). Les idées visuelles ne renvoient que dérivativement à des idées spatiales. Ce renvoi à des idées spatiales n'est possible qu'au terme d'un apprentissage au cours duquel nous avons appris à associer à certaines qualités de l'expérience visuelle ainsi qu'aux sensations de convergence et d'accommodation diverses idées spatiales (de forme, de taille, de distance) qui ont leur origine dans le toucher.

Berkeley donne deux justifications du privilège accordé au toucher vis-à-vis de la vision dans la connaissance spatiale. En premier lieu, les idées tactiles ont l'avantage de la stabilité. Les idées tactiles de la forme et de la taille des objets extérieurs sont constantes, alors que les idées visuelles qui sont associées à un objet sont fluctuantes. Cette invariance est une condition de possibilité d'un discours libre d'ambiguité sur la réalité. Deuxièmement, notre intérêt pour la réalité est pour Berkeley avant tout pragmatique. La réalité nous intéresse pour autant qu'elle affecte notre bien-être et donc influe sur notre comportement. Or nos sensations de plaisir et de peine dépendent de nos rencontres avec les qualités tangibles des corps extérieurs, non avec leurs qualités visibles. Nous sommes donc intéressés essentiellement par les qualités tangibles des objets et le sens de la vue a pour utilité principale de nous permettre d'anticiper ces qualités :

le sens de la vue semble avoir été accordé aux animaux [...] pour que, par la perception des idées visuelles (qui, en elles-mêmes, ne sont pas capables d'affecter ou de modifier d'une façon quelconque la conformation de leur corps), ils puissent être capables de prévoir (d'après l'expérience qui leur a appris que telles idées

3 Cette section prendra appui sur les analyses de Morgan (1977) et d'Evans (1985) ainsi que sur I'analyse remarquable de darté que donne Robert Schwartz (1994) des thèses défendues par Berkeley dans son Essai pour une nouvelle théorie de la vision (1709). 
tactiles sont associées à telles et telles idées visuelles) le dommage ou le bénéfice qui sont à même de s'ensuivre au contact de leurs propres corps avec tel ou tel corps qui est à distance. $(1710, \S 59$, p. 230)

On voit donc que ce qui intéresse principalement Berkeley, en tout cas lorsqu'il écrit l'Essai, ce ne sont pas les qualités phénoménales des différentes modalités perceptives, mais les informations sur la réalité qui peuvent ou non nous être directement données par l'une ou l'autre modalité. Pour Berkeley, seul le toucher nous donne un accès direct au réel.

A la suite de Schwartz (1994), Il importe de préciser le sens de l'opposition entre idées médiates et immédiates telle qu'elle est utilisée par Berkeley et ses contemporains. Cette opposition est liée aux types de processus par lesquels ces idées sont produites dans l'esprit. Sont immédiates, au sens de Berkeley, les idées qui se produisent dans l'esprit directement au terme d'un processus purement physiologique ou organique, sans que la médiation de processus mentaux ou psychologiques soit nécessaire pour leur production. Elles correspondent à ce que Helmholtz appellera plus tard les sensations, définies comme la contribution faite à notre expérience par les organes des sens préalablement à toute élaboration par l'esprit, à l'étude desquelles il consacrera le second volume de son célèbre Traité d'optique physiologique. Inversement, sont médiates les idées dont la production exige l'intervention de processus psychologiques ou mentaux. L'opposition entre idées immédiates et médiates correspond donc sans doute à l'un des sens de l'opposition entre sensations et perceptions 4 Pour Berkeley, un processus est mental ou psychologique pour autant qu'il fait intervenir la manipulation d'idées, elles-mêmes conçues comme des états de conscience (possibles). Toutefois, le fait qu'une idée, médiate ou immédiate, soit un état de conscience n'implique nullement pour lui l'incorrigibilité de nos rapports introspectifs sur ces idées : il n'est pas assuré que nous puissions les ressaisir distinctement et correctement par l'introspection. Nous pouvons nous tromper sur la nature et la qualité des idées immédiates des sens. Il est notamment possible d'expliquer l'impression générale quasi-irrésistible selon laquelle il y a quelque chose d'intrinséquement spatial dans notre expérience visuelle par la quasiimpossibilité où nous sommes de séparer dans notre esprit les objets de la vue et du toucher qui ont été intimement associés depuis notre plus tendre enfance.

${ }^{4}$ Cette manière de tracer la distinction ne doit pas être confondue avec une autre manière également traditionnelle de distinguer sensations et perceptions, qui insiste sur le fait que les perceptions ont un contenu représentationnel, représentent l'environnement comme étant tel et tel, alors que les sensations sont simplement des états qualitatifs du sujet lui-même. Pour une analyse des rapports entre les deux distinctions, cf. Hatfield, 1990, Appendice B. 
Enfin, est encore lié à la distinction entre idée immédiates et médiates un autre présupposé très important, quoique implicite, partagé par Berkeley et ses contemporains. A la distinction entre idées immédiates et médiates, produits d'un processus purement physiologique et produits d'un processus ou interviennent des opérations proprement mentales, fait exactement écho la distinction entre processus non susceptibles d'être modifiés par l'apprentissage et processus susceptibles de l'être. Comme le souligne Schwartz, "il était très commun de soutenir [...] que l'apprentissage ne pouvait avoir d'effet sur nos organes sensoriels, que les productions des sens étaient fixées physiologiquement. Les réponses des sens pouvaient être altérées par la maladie et la fatigue mais non par l'expérience. On supposait donc que tout phénomène perceptif qui manifestait l'influence de l'apprentissage ne pouvait être immédiat, un aspect de la sensation, mais devait être non-immédiat, résultant de l'élaboration de la sensation par des opérations mentales." (1994 : 12). Ce présupposé de la fixité des sensations explique que la quasi-totalité des philosophes qui se sont intéressés au XVIIIe siècle à la question de Molyneux, aient admis que l'aveugle nouvellement opéré verrait, au sens où il aurait des sensations visuelles par hypothèse semblables à celles des autres hommes.

On peut être tenté de tenir Diderot pour une voix discordante dans ce concert philosophique. Dans la Lettre aux Aveugles, il élève en effet des doutes sur la question de "savoir si un aveugle-né auquel on abattrait les cataractes, serait en état de se servir de ses yeux dans les premiers moments qui succèdent à l'opération" (1749: 846-847), question qu'il tient pour un préliminaire à la question de Molyneux. Toutefois, à y regarder de plus près, il semble que les réserves émises sur ce chapitre par Diderot ne soient pas motivées par l'idée qu'un apprentissage des sensations elles-mêmes est nécessaire, mais concernent plutôt, en amont des sensations, l'état des organes de la vue et leur capacité à remplir immédiatement leur fonction 5 et en aval de celles-ci, la nécessité de l'expérience - mais pas nécessairement d'un apprentissage guidé par le toucher - pour analyser nos sensations et les rapporter au monde.

5 Diderot mentionne en particulier "le temps nécessaire aux humeurs de l'œil pour se disposer convenablement; à la cornée pour prendre la convexité requise à la vision ; à la prunelle, pour être susceptible de la dilatation et du rétrécissement qui lui sont propres ; aux filets de la rétine, pour n'être ni trop ni trop peu sensibles à l'action de la lumière; au cristallin, pour s'exercer aux mouvements en avant et en arrière qu'on lui soupçonne; ou aux muscles, pour bien remplir leurs fonctions; aux nerfs optiques pour s'accoutumer à transmettre la sensation; au globe entier de l'œil, pour se prêter à toutes les dispositions nécessaires, et à toutes les parties qui le composent, pour concourir à l'exécution de cette miniature. (1749: 852-853) 
La thèse essentielle sur laquelle repose la réponse négative de Berkeley à la question de Molyneux est que l'expérience visuelle - autrement dit les sensations visuelles - n'a en elle-même aucun contenu spatial. En termes plus berkeleyiens, les idées d'étendue, de figure et de mouvement ne constituent pas l'objet propre et immédiat de la vue, puisque "tout ce qui est proprement perçu par la faculté visuelle se réduit aux seules couleurs, à leurs variations et aux différents degrés d'ombre et de lumière" (1985, § 156, p. 280). On notera qu'il s'agit là d'une thèse beaucoup plus forte que la thèse, communément acceptée à l'époque, selon laquelle nous ne percevons pas immédiatement la distance. Cette thèse plus faible laisse ouverte la possibilité que l'objet immédiat de la perception visuelle soit une image bi-dimensionnelle à partir de laquelle nous inférerions des propriétés tri-dimensionnelles non directement données. Mais, pour Berkeley, l'objet immédiat de la perception visuelle est dépourvu de toute spatialité intrinsèque. Ainsi qu'il y insiste dans les dernières sections de l'Essai, "les plans ne sont pas plus que les solides l'objet immédiat de la vue. Ce que nous voyons au sens strict, ce ne sont pas des solides, ni même des plans diversement colorés, mais c'est seulement une diversité de couleurs" (1985, §158, p. 281). Comme le fait remarquer Schwartz (1994 : 29), cela ne signifie pas que le champ visuel n'est pas pas structuré, mais simplement que son mode de structuration n'est pas spatial. 6

La comparaison qu'établit le dernier paragraphe de l'Essai avec le langage est à maints égards éclairante : (1) de même que les "sons d'une langue" n'ont en eux-mêmes aucune connexion nécessaire avec les significations qui leurs sont attachées, de mêmes les idées purement visuelles n'ont en elles-mêmes aucune connexion nécessaire avec les idées spatiales; (2) de même, par conséquent, que c'est seulement au terme d'un apprentissage que nous associons à des sons donnés des significations déterminées, de même c'est seulement au terme d'un apprentissage que nous associons aux perceptions visuelles des idées spatiales; (3) de même que les règles de structuration du langage n'ont pas à être de même nature que les règles et lois qui gouvernent les relations entre les entités signifiées par le moyen du langage, de même le mode de structuration de nos perceptions visuelles n'a pas à être de même nature que le mode de structuration (géométrique) des propriétés spatiales; et enfin (4) de même qu'il peut être

6 L'opposition soulignée par Berkeley entre le caractère constant des grandeurs tangibles et, au contraire, le caractère fluctuant et indéterminé des grandeurs visibles me conduit à hasarder I'hypothèse que, pour Berkeley, la notion de grandeur absolue est un ingrédient essentiel de toute idée proprement spatial e et que c'est précisément l'absence de cet ingrédient dans les idées immédiates de la vue qui motive en profondeur la thèse Berkeleyienne selon laquelle les idées visuelles ne sont pas à strictement parler spatiales. 
extrêmement difficile voire impossible à quelqu'un "d'entendre prononcer à ses oreilles les mots de sa langue maternelle sans les comprendre [... et] de séparer le sens du son" (1985, §159, p. 182), de même, il peut être quasi-impossible de séparer les idées visuelles des idées spatiales auxquelles elles ont depuis le plus jeune âge été associées via le toucher. Donc, pour en revenir au problème de Molyneux, demander si l'aveugle recouvrant la vue serait capable sans les toucher de distinguer le cube et la sphère est équivalent, du point de vue de Berkeley, à demander si une personne entendant pour la première fois les mots servant à désigner les cubes et les sphères dans une langue qu'il ignore, saurait immédiatement quel mot désigne quoi.

On attribue souvent la réponse négative de Berkeley à la question de Molyneux à son refus des idées abstraites et à son anti-innéisme. Ces deux considérations entrent certainement pour une part dans la prise de position de Berkeley, mais à mon sens interviennent plutôt à titre auxiliaire. Essayons de préciser leur rôle. Pour que la thèse berkeleyienne du caractère strictement non-spatial des idées immédiates de la vision conduise nécessairement à une réponse négative à la question de Molyneux, il faut que soient préalablement exclues certaines autres possibilités. C'est là, à mon sens, que l'anti-innéisme et l'anti-abstractionnisme de Berkeley jouent un rôle stratégique. On pourrait par exemple imaginer que quoique les modalités visuelles et tactiles n'aient aucune qualité en commun et que la perception des propriétés spatiales constitue le domaine propre du toucher, il existe néanmoins une corrélation systématique innée entre sensations tactiles et visuelles. Cette corrélation pourrait être soit directe (faisant intervenir une forme de synesthèsie) soit indirecte, opérant par la médiation d'une idée abstraite supra-sensible. Il serait alors possible à l'aveugle nouvellement opéré de discerner le cube et la sphère sans qu'aucun apprentissage des corrélations entre la vue et le toucher ne lui soit nécessaire. La première possibilité - l'existence d'une corrélation directe entre sensations du toucher et sensations de la vue - n'est pas envisagée par Berkeley. On peut penser en effet qu'accepter cette corrélation conduit à nier la thèse de l'hétérogénéïté des idées immédiates de la vue et du toucher. Faire l'hypothèse d'une corrélation directe innée entre sensations tactiles et sensations visuelles revient à postuler l'existence d'un mécanisme physiologique assurant la corrélation. Mais dans ce cas, des sensations tactiles, et ainsi des idées spatiales, constitueraient l'objet immédiat de l'expérience visuelle, ce qui n'est rien d'autre que la négation pure et simple du postulat fondamental de Berkeley. 7

7 Dans une perspective plus contemporaine, on notera toutefois qu'il est en principe possible de distinguer empiriquement entre amodalité et corrélations systématiques innées entre modalités distinctes 
On notera ici, qu'à supposer même que Berkeley eût discuté cette possibilité et l'eût refusé, ce refus n'eût pas été en soi la marque d'un anti-innéisme. Il importe en effet de ne pas se laisser abuser par le sens aujourd'hui communément attaché à la notion d'innéité dans les débats de la psychologie et de bien identifier ce que combattaient au juste les empiristes du XVIIIème siècle lorsqu'ils s'en prenaient aux idées innées. Ainsi que le fait remarquer Morgan (1977), la plupart des empiristes du XVIIIle siècle seraient aujourd'hui considérés comme des innéistes en ce sens qu'ils admettaient parfaitement que les propriétés qualitatives des sensations excitées en nous à l'occasion de certaines formes de stimulations étaient déterminées par notre nature ou, ce qui revient au même, instituées par Dieu; en d'autres termes, ils admettaient que la nature de certaines des sensations éveillées en nous par différents objets dépend de dispositions innées et, ainsi que nous l'avons déjà mentionné, non-modifiables par l'expérience. L'opposition des empiristes à l'innéisme ne visait donc nullement l'existence de telles dipositions innées (d'un pouvoir inné de sentir), mais la thèse selon laquelle des idées pourraient être présentes dans notre esprit préalablement à tout usage de ces dispositions. En d'autres termes, pour reprendre l'exemple de Hume, cité par Morgan (1977 : 83), quoique la nature de la sensation que nous pourrons éprouver en goûtant un ananas soit déterminée de manière innée, nous ne saurions avoir une idée juste du goût d'un ananas avant d'en avoir effectivement goûté.

En fin de compte, si Berkeley avait envisagé la possibilité d'une corrélation innée entre sensations visuelles et sensations tactiles, il ne lui aurait pas été nécessaire pour l'écarter, de faire appel à des postulats anti-innéistes; il lui aurait suffit d'affirmer l'absence de fait d'une telle corrélation. En revanche, l'anti-innéisme et l'antiabstractionnisme de Berkeley interviennent dans la mise à l'écart de la seconde possibilité, celle d'une corrélation indirecte des sensations de la vue et du toucher par l'intermédiaire d'idées supra-sensibles se surordonnant à elles. Les idées de la vue et du toucher n'ayant rien de commun, une idée qui subsumerait les unes et les autres ne saurait résulter d'un processus de généralisation exploitant leurs similitudes ; elle devrait nécessairement être innée et abstraite.

La raison pour laquelle l'attitude vis-à-vis des idées innées et des idées abstraites n'est toutefois pas l'enjeu principal de la question de Molyneux est qu'il est parfaitement possible d'apporter une réponse positive à cette question sans devoir pour cela admettre l'existence de telles idées. On peut le faire en affirmant, contre Berkeley, l'existence d'une corrélation directe innée entre les sensations du toucher et celles de la

sur la base des dysfonctionnements différents, congénitaux ou acquis, dont sont susceptibles les deux types d'organisation. 
vue, ou bien encore, ce que refuserait également Berkeley, en supposant l'existence d'un recoupement au moins partiel entre les sensations visuelles et les sensations tactiles, tel que les idées spatiales soient communes aux deux sens. En termes contemporains, ceci reviendrait à affirmer, contrairement à ce que soutient Berkeley, que les sensations visuelles et tactiles ne sont pas définies sur des espaces de qualités totalement hétérogènes et disjoints, mais qu'elles s'inscrivent dans le même espace général de qualités et prennent l'une et l'autre des valeurs sur les dimensions spatiales de cet espace.

Nous en avons dit assez pour pouvoir commencer à dessiner une typologie des motivations susceptibles de sous-tendre les réponses tant négatives que positives au problème de Molyneux. La typologie que je propose recoupe pour une grande part la typologie proposée par Evans (1985) qui considère que la question principale soustendant le problème de Molyneux concernait l'existence d'une spatialité inhérente à la perception visuelle. Je me démarque toutefois d'Evans sur deux points. Je ne prends pas en considération la thèse selon laquelle les aveugles ne possèdent pas de véritables concepts spatiaux. D'une part, en effet, cette position ne semble pas avoir joué un grand rôle dans le débat qui a traversé le XVIIIème siècle8 D'autre part, je partage l'opinion d'Evans (1985) et Millikan (1993) selon laquelle cette thèse repose sur une confusion consistant à projeter dans le contenu représenté les propriétés du véhicule représentationnel. En l'occurrence, l'erreur consiste à soutenir que l'aveugle ne saurait posséder de concepts spatiaux véritables — qui font intervenir l'idée de l'existence simultanée d'objets distincts - parce que l'appréhension des objets par les sens autres que la vue est successive et non simultanée. Ma seconde modification de la typologie d'Evans consistera à greffer une nouvelle branche sur son arbre pour faire place à certaines considérations avancées par Diderot et par Condillac, non par un simple souci historique d'exhaustivité, mais parce que ces considérations trouvent leur écho dans des interrogations qui sont devenues centrales dans la problématique contemporaine.

Si l'on considère que le problème de Molyneux a pour enjeu essentiel l'existence d'une spatialité inhérente à la perception visuelle, on pourrait être tenté de croire qu'une réponse négative à la question de la spatialité de la perception visuelle entraîne nécessairement une réponse négative au problème de Molyneux et qu'à l'inverse une réponse positive à la première question entraîne une réponse positive au problème de Molyneux. C'est notamment ce que pourrait suggérer la position de Berkeley, qui, ainsi

\footnotetext{
${ }^{8}$ Le plus récent avocat de cette position est M. Von Senden (1960) . D'après les recherches menées par Evans, le premier auteur qui ait explicitement énoncé cette position est Platner dans ses Philosophische A phorismen (1793)
} 
que nous l'avons vu, niait que les idées immédiates de la vue possédent une spatialité intrinsèque et, sur cette base, répondait négativement à la question de Molyneux. Nous devons toutefois résister à cette interprétation tentante. En premier lieu, même en s'accordant avec Berkeley pour nier l'existence d'une spatialité intrinsèque à la vision, on peut encore avancer au moins trois raisons d'apporter une réponse positive au problème de Molyneux. Comme nous l'avons déjà vu, on peut soutenir qu'il existe une connexion immédiate innée entre les sensations tactiles et les sensations visuelles; on peut également soutenir qu'il existe une connexion innée entre sensations visuelles et sensations tactiles via l'intermédiaire d'idées abstraites qui les subsument les unes et les autres. Enfin, l'on peut dire avec Leibniz et avec le géomètre de Diderot que l'aveugle nouvellement opéré soumis au test devrait pouvoir aboutir à la bonne réponse par le raisonnement, en exploitant le fait que "dans le globe il n'y a pas de points distingués du côté du globe même, tout y étant uni et sans angles, au lieu que dans le cube, il y a huit points distingués de tous les autres" (Leibniz, 1765, II, IX, p. 114). Leibniz précise toutefois que pour que l'aveugle-né use de cet expédient, il faut qu'il ait été instruit préalablement de ce qu'il a devant lui un cube et une sphère, sans quoi il ne s'aviserait pas spontanément de faire le lien entre les impressions visuelles qu'il reçoit et ces idées. Quant au géomètre de Diderote il est conscient de ce que son raisonnement repose sur la condition que les impressions de la vue et du toucher ne soient pas contradictoires et il tient que cette condition est remplie du fait que ses auditeurs voyants comprenaient en voyant les figures les démonstrations qu'il faisait de leurs propriétés sur la base de ses impressions tactiles. On notera accessoirement que Berkeley lui-même accordait cette possibilité, et reconnaissait "que le carré visible est plus apte à représenter le carré tangible" que le cercle visible, mais insistait sur le fait que cette aptitude représentationnelle ne se fondait nullement sur une ressemblance ; en outre, il serait en principe assez facile de créer la perplexité chez l'aveugle en lui présentant d'une part un carré de couleur unie, d'autre part un cercle comportant quatre quartiers diversement colorés.

$\mathrm{Si}$, au contraire de Berkeley, on soutient qu'il existe une spatialité intrinsèque à la vision, il semblerait que l'on s'engage par là à répondre positivement à la question de Molyneux. Cependant, il reste possible, même dans ce cas, d'apporter une réponse négative. Il est vrai qu'aucun des philosophes du XVIIIème siècle n'a expressément adopté la position que je vais décrire. On peut toutefois trouver chez Condillac, même s'il s'est rangé à l'avis de Berkeley, des considérations sur lesquelles on peut appuyer

9 Le géomètre de Diderot a pour modèle le mathématicien aveugle de Cambridge, Saunderson, longuement évoqué dans la Lettre sur les aveugles. 
cette position. C'est en fait Diderot, inspiré sur ce point par les idées de Condillac, qui est passé le plus près de cette réponse.

Rétrospectivement, l'un des grands mérites de Condillac est d'avoir mis l'accent sur le caractère actif de la perception et d'avoir souligné qu'il ne suffit pas de voir pour connaître mais qu'il faut encore regarder :

Il ne suffit pas de répéter, d'après Locke, que toutes nos connaissances viennent des sens: si je ne sais pas comment elles en viennent, je croirai qu'aussitôt que les objets font des impressions sur nous, nous avons toutes les idées que nos sensations peuvent enfermer, et je me tromperai. [...] Il semble qu'on ne sache pas qu'il y a de la différence entre voir et regarder; et cependant nous ne nous faisons pas des idées, aussitôt que nous voyons; nous ne nous en faisons qu' autant que nous regardons et que nous regardons avec ordre, avec méthode. En un mot, il faut que nos yeux analysent : car ils ne saisiront pas l'ensemble de la figure la moins composée, s'ils n'en ont pas observé toutes les parties, séparément, l'une après l'autre, et dans l'ordre où elles sont entre elles. (1754, III, III, p. 170)

L'un des objets principaux de Condillac était de répondre à la question suivante : si toute notre connaissance du monde nous vient de nos sensations, et si toutes les sensations sont simplement des modifications de notre esprit, comment pouvons-nous justifier l'existence du monde externe ? Ainsi que le souligne Morgan (1977 : 69), cette question en recouvre en fait deux: la question proprement épistémologique de la justification de notre croyance au monde externe et la question plus psychologique consistant à expliquer comment il se fait que nous croyons à l'existence d'un monde externe doté d'une certaine organisation spatiale. Alors que l'on peut penser que l'immatérialisme de Berkeley constitue une réponse à la question épistémologique, les considérations déroulées par Condillac dans le Traité des Sensations visent plutôt à répondre à la seconde question. En particulier, la distinction entre un voir passif et une regarder actif est mobilisée à cet effet. Nous nous représentons le monde pour autant que nous interagissons avec lui. Si on ne peut se représenter l'existence d'un monde extérieur sans se représenter cette existence comme spatiale, autrement dit, si objectivité et spatialité vont de pair et si, comme le veut Condillac, la représentation d'objets comme existants dans le monde externe suppose un processus actif de perception, alors il ne suffit pas de voir, de recevoir passivement des sensations, pour distinguer la sphère comme telle $\mathrm{du}$ cube, il faut encore analyser ces sensations. Autrement dit, ce sont les mouvements exploratoires et notamment les déplacements oculaires qui interviennent dans l'analyse des sensations qui permettent de conférer à celles-ci une organisation spatiale. L'aveugle nouvellement opéré qui recevrait 
passivement des sensations visuelles ne saurait donc distinguer la sphère du cube. En outre, dans la mesure où analyser nos sensations n'est pas quelque chose que nous sachions spontanément faire, il faut à l'aveugle le temps d'apprendre à regarder et observer correctement.

Toutefois, la raison dernière pour laquelle Condillac répond négativement à la question de Molyneux n'est pas qu'il faut du temps pour apprendre à regarder, mais plutôt que la reconnaissance de l'existence d'un monde extérieur et l'exploration active de l'environnement dépendent d'une expérience préalable du toucher (ce qui chez Condillac inclut la proprioception) et du mouvement. En premier lieu donc, le toucher est le "seul sens qui juge par lui-même des objets extérieurs" (1754, II, p. 89), c'est-àdire, pour Condillac, qui nous donne par lui-même l'idée qu'existent hors de nous des corps étendus. Par elles-mêmes, les sensations visuelles n'apparaissent que comme des modifications de l'âme et ne donnent pas l'idée d'un monde extérieur. Condillac insiste sur deux propriétés des corps qu'il juge essentielles : la première est que les corps sont des continus formés par la contiguïté d'autres corps étendus; la seconde est qu'ils sont impénétrables, au sens où deux corps ne sauraient occuper le même lieu. L'idée d'impénétrabilité nous est donnée à travers la sensation de solidité que nous éprouvons au contact d'un corps solide. Cette sensation de solidité, nous l'éprouvons d'abord en touchant notre propre corps et en nous éprouvant ainsi à la fois comme touchant et touché. C'est parce que "le propre d'une telle sensation est de représenter à la fois deux choses qui s'excluent l'une hors de l'autre"(1754, II, p. 103) que l'âme ne pourra la percevoir comme une simple modification d'elle-même et devra former l'idée de quelque chose hors d'elle-même. Toutefois, pour que l'âme ait pleinement l'idée de l'étendue, il lui faut encore acquérir l'idée de la connexité des corps étendus. C'est ici qu'intervient le mouvement, car c'est seulement en se déplaçant le long du corps que la main réunit en un seul continu les différentes sensations de solidité éprouvées en touchant différentes parties du corps.

Dans le passage du voir au regarder, nécessaire à la constitution d'une représentation visuelle d'un monde extérieur, l'expérience préalable du toucher joue un rôle capital. D'abord, nous venons de le voir, elle est à l'origine de la distinction entre l'esprit et ses modifications, d'une part, et un monde extérieur, d'autre part. Ensuite, pour connaître par la vue des objets du monde externe, il faut non seulement observer, il faut observer avec ordre et méthode. Mais si une exploration ordonnée est un prérequis pour une expérience spatiale visuelle, l'ordre en question ne peut être dérivé de l'expérience purement visuelle ; il doit être prédonné. Pour Condillac, il l'est dans la proprioception, en tant que mode de connaissance des mouvements de notre propre corps. La proprioception est donc condition de l'appréhension de l'expérience visuelle 
comme spatialement ordonnée. Apparaît ici centralement le thème du rôle du mouvement dans la perception spatiale. Pour Condillac, il ne saurait y avoir de perception proprement spatiale sans mouvement. Or le mouvement ici considéré est le mouvement de l'observateur, intrinsèquement lié, pour Condillac, à la proprioception, d'où une version sophistiquée de la thèse que la vision est éduquée par le toucher.

Il n'est pourtant pas certain qu'une conception "active" de la perception visuelle spatiale doive conduire à l'idée d'une dépendance nécessaire de la perception visuelle spatiale vis-à-vis du toucher. Une autre option est esquissée par Diderot dans la Lettre aux Aveugles. Diderot convient, dans un esprit tout à fait condillacien, que l'expérience est nécessaire à la vision, "que nous [les adultes voyants] devons apercevoir dans les objets une infinité de choses que l'enfant ni l'aveugle-né n'y aperçoivent point, quoiqu'elles se peignent également au fond de leurs yeux ; que ce n'est pas assez que les objets nous frappent, qu'il faut encore que nous soyons attentifs à leurs impressions ; que par conséquent, on ne voit rien la première fois que l'on se sert de ses yeux; qu'on est affecté dans les premiers instants de la vision, que d'une multitude de sensations confuses qui ne se débrouillent qu'avec le temps et par la réflexion habituelle sur ce qui se passe en nous [...]" (1749: 850-851). Diderot convient également de ce que, dans les faits, le toucher joue un grand rôle dans l'instruction de l'œil. Mais il refuse en revanche d'admettre qu'en principe il ne puisse en aller autrement: "Cependant, je ne pense nullement que l'œil ne puisse s'instruire, ou, s'il est permis de parler ainsi, s'expérimenter de lui-même. Pour s'assurer par le toucher, de l'existence et de la figure des objets, il n'est pas nécessaire de voir ; pourquoi faudrait-il toucher, pour s'assurer des mêmes choses par la vue ? [...] On conçoit sans peine que l'usage d'un des sens peut être perfectionné et accéléré par les observations de l'autre ; mais nullement qu'il y ait entre leurs fonctions une dépendance essentielle" (1749: 851). Les considérations que Diderot avance dans la Lettre sur les Aveugles en faveur de cette position ne sont pas décisives. Toutefois, Diderot a le mérite de mettre en évidence une nouvelle option relativement au problème de Molyneux : celle d'une réponse négative qui ne reposerait pas sur le déni d'une spatialité intrinséque à la vision, mais sur l'idée que, pour qu'il puisse y avoir perception spatiale, l'œil doit d'abord s'instruire, même s'il peut en principe le faire en autodidacte.

\section{Le problème de Bach-y-Rita ou l'indépendance des sensations et des perceptions}

Le débat contemporain sur le problème de Molyneux interprète celui-ci comme portant sur le caractère modal ou amodal de la perception spatiale et de ce point de vue prolonge directement le débat des Lumières. Ce n'est pas dire toutefois que tous les 
auteurs s'accordent sur une même interprétation de l'enjeu exact de la question de Molyneux, puisque Eilan (1993a) ne distingue pas moins de dix sens possibles de la distinction entre amodalité et spécificité modale, ceci sans nullement prétendre à l'exhaustivité. On ne saurait toutefois se plaindre de cette abondance d'acceptions, car il semble que ce soit le défaut de quelques-unes de ces distinctions qui ait rendu possible certaines des réponses négatives apportées au XVIIIème siècle à la question de Molyneux. Je vais maintenant présenter une variante moderne du problème de Molyneux que j'appelerai problème de Bach-y-Rita et qui présente plusieurs avantages dont celui de rendre manifeste la nécessité d'une distinction entre deux thèses de spécificité modale et celui d'illustrer positivement l'option esquissée par Diderot.

Dans la deuxième moitié des années 1960, Bach-y-Rita et ses collègues (Bach-yRita et al., 1969 ; White et al., 1970, Bach-y-Rita, 1972, Collins et al., 1973) ont mis au point un système de substitution visuo-tactile (SSVT) destiné aux aveugles. Ce système est constitué en premier lieu d'une caméra vidéo, que le sujet peut manipuler à sa guise. Cette caméra est couplée à un mécanisme de transduction, qui convertit l'image produite par la caméra en une représentation cutanée isomorphe sous forme d'une configuration de vibrations produite par une matrice de 400 vibrateurs placée sur la peau du sujet, généralement dans le dos ou sur l'abdomen. Avant de présenter les résultats des expériences menées avec ce système, il importe de souligner certaines différences par rapport au problème de Molyneux dans sa version originale et les avantages qu'elles présentent.

Comme le souligne Morgan (1977), le problème de Molyneux dans sa version originale présente de graves inconvénients pratiques. L'idée que l'étude des aveugle-nés venant de recouvrer la vue puisse nous éclairer sur la caractère spatial ou non-spatial de la perception visuelle repose sur l'hypothèse plus que douteuse que l'équipement sensoriel de l'aveugle congénital soit intact et prêt à fonctionner au premier instant de la vision. Faute de garantie sur ce point, il est impossible de déterminer si l'aveugle qui ne discerne pas le cube de la sphère ne le fait pas parce que l'information visuelle qu'il obtient par les yeux ne porte pas sur les propriétés spatiales de l'environnement ou bien parce que son système sensoriel, fonctionnant mal, n'est pas capable d'obtenir cette information spatiale 10

Un des grands avantages du SSVT est de court-circuiter cette difficulté en rendant disponible au sujet une information essentiellement visuelle sans avoir à passer par les

${ }^{10}$ En fait, les études modernes ont montré que les aveugles-nés opérés conservent (surtout s'ils ont été opérés tardivement) un déficit visuel persistant qui leur interdit la discrimination des formes. Voir sur ce sujet Jeannerod (1975). 
yeux. Dans le cas de l'utilisation du SSVT comme dans celui de la vision ordinaire, l'information qui arrive au sujet est l'information sur les objets véhiculée par la lumière ; et dans les deux cas cette information est présentée et extraite de la même manière. Morgan relève par exemple la liste suivante, non exhaustive, de similitudes :

(a) Une image est formée par une lentille sur une surface bi-dimensionnelle.

(b) La surface contient des éléments discrets (vibrateurs ; bâtonnets et cônes) qui réagissent à l'entrée.

(c) Les surfaces contiennent des cellules nerveuses, qui sont connectées aux régions de la surface ("champs récepteurs") et qui envoient des signaux électriques au cerveau.

(d) Le système de formation de l'image (œil ; caméra de télévision) peut être déplacé à volonté, soumettant l'image à des transformations.

(e) Plus généralement, dans les deux systèmes la source de stimulation n'est pas nécessairement en contact avec le corps ; autrement dit, elle peut être sentie, même si elle ne peut pas nécessairement être touchée.

(f) En conséquence de (e) la perception peut être interrompue par l'interposition d'objets entre le spectateur et l'objet vu. (Morgan, 1977 : 201)

Comme Morgan le souligne encore, la différence entre les deux systèmes est d'ordre plus quantitatif que qualitatif : il y a beaucoup moins de récepteurs dans le SSVT que dans l'œil, de même qu'il y a beaucoup moins de fibres nerveuses allant de la zone de la peau qui se trouve sous la matrice de vibrateurs qu'il n'y a de fibres nerveuses dans le nerf optique. Mais ces types de différences quantitatives se rencontrent aussi entre les différentes espèces animales. Il y a certes une autre différence plus qualitative entre les deux systèmes qui tient à ce que le SSVT ne permet pas de voir les couleurs, mais nous en réservons la discussion pour plus tard. Un autre avantage de l'utilisation de SSVT est qu'elle permet le contrôle et l'évaluation de tous les aspects de l'apprentissage visuel, puisque les sujets n'ont d'expérience visuelle que lorsque l'appareillage est porté.

Mettons fin au suspense. Que se passe-t-il lorsque des aveugles sont équipés du SSVT ? Ils deviennent très rapidement - au terme de 5 à 15 heures de pratique en moyenne - capables de distinguer des objets et de décrire leur arrangement spatial, d'évaluer correctement la taille, la distance, la rotation, l'orientation et autres phénomènes tri-dimensionnels qui sont présentés. Ils réagissent par un comportement de défense à l'approche soudaine d'un objet dans le champ de la caméra. Ils répondent aux illusions visuelles qui ont été testées (par exemple, l'effet de cascade) de la même façon que les voyants. Au bout de quelques heures de pratique, les stimuli perçus sont localisés par le sujet dans le monde extérieur, en face de lui, et non au contact de la 
peau, autrement dit, ils sont perçus comme distaux. Les sensations proprement cutanées sont ignorées sauf en cas de gêne. La progression type au cours de l'apprentissage semble la suivante. Certains arrangements simples sont presque immédiatement perçus : les lignes horizontales et verticales sont distinguées et correctement identifiées; le mouvement d'une bande verticale se déplaçant d'un côté à l'autre peut être correctement décrit et imité avec un geste de la main. L'identification des objets est moins immédiate et opère principalement sur la base d'une inspection de leurs contours. Lors des premières présentations, l'identification peut prendre plusieurs minutes mais est ramenée à quelques secondes après plusieurs heures de pratiques (de 2 à 20 secondes après 10 heures de pratique). Dans les premiers temps, l'évaluation de la distance et de la profondeur fait intervenir l'utilisation consciente de certains indices tels que la familiarité avec les objets ou l'interposition. Avec la pratique, ces jugements deviennent plus ou moins automatiques. On notera enfin la grande importance du mouvement et notamment du mouvement contrôlé par le sujet, à la fois dans la reconnaissance des formes et dans la localisation externe des stimuli :

Les résultats obtenus jusqu'ici indiquent la grande importance des mouvements auto-générés de l'observateur. Lorsqu'il leur est demandé d'identifier des formes statiques avec la caméra fixe, les sujets éprouvent de grandes difficultés ; mais quand ils sont libres de tourner la caméra pour explorer les figures, la discrimination est rapidement établie. Avec la caméra fixe, les sujets rapportent leurs expériences en termes de sensations sur le dos, mais quand ils déplacent la caméra sur les agencement\$11 ils rapportent leur expérience en termes d'objets localisés extérieurement en face d'eux. Le mouvement de la caméra est ici analogue au mouvement des yeux dans la vision et ce résultat soulève la possibilité intéressante que la localisation externe des percepts puisse dépendre, de manière critique, de ces mouvements." (White et al., $1970: 25$ )

Quelle leçon tirer de ces résultats vis-à-vis du problème de Molyneux ? J'ai déjà indiqué qu'ils me semblaient aller dans le sens de l'option suggérée par Diderot, à savoir que la vision nous donne par elle-même des informations sur les propriétés spatiales de l'environnement, mais qu'elle a néanmoins besoin pour ce faire d'un apprentissage. Pour que cette conclusion ait quelque apparence de vraisemblance, il faut d'abord montrer (1) qu'il est raisonnable d'attribuer à l'utilisateur du SSVT une expérience visuelle et (2) que, si important que soit le rôle du mouvement dans la perception

\footnotetext{
11 Le mot anglais est “layout” qui désigne la manière dont les objets sont disposés dan l'espace.
} 
visuelle, la reconnaissance de ce rôle n'équivaut pas à la reconnaissance du caractère dérivé de l'espace visuel par rapport à l'espace tactile.

Le SSVT est une prothèse sensorielle, mais de quoi est-elle la prothèse ? Quel est le sens qu'elle étend ou à la défaillance duquel elle supplée ? J'ai déjà noté, à la suite de Morgan, la similitude informationnelle entre le processus normal de la vision et le processus qui intervient avec le SSVT. Les récits faits par les sujets qui ont utilisé le SSVT témoignent également d'une similitude représentationnelle. L'expérience perceptive est éprouvée comme étant celle d'un monde externe stable et les objets sont perçus comme étant à distance du sujet et non en contact immédiat avec lui. Toutefois, le SSVT fait intervenir deux types de stimulations physiques: d'abord les rayons lumineux sur la lentille de la caméra et, ensuite, les vibrations mécaniques sur la peau. Etant donné cette intervention des stimulations tactiles dans le processus, n'avons-nous pas affaire à une forme de perception tactile ? Cette objection est envisagée par Heil (1983) qui y répond en faisant remarquer que "dans la mesure où l'on veut décrire l'utilisateur du SSVT comme faisant usage de son sens du toucher, les objets sentis ne sont pas ceux qui se trouvent en face de la caméra de télévision, mais les vibrateurs mis au contact de sa peau. $\mathrm{Si}$, au contraire, on considère que les objets qu'il sent sont ceux qui sont balayés par la caméra, il est certainement plus plausible de les décrire comme (en un sens) vus, les croyances ainsi acquises comme (en un sens) visuelles" (1983: 16). On peut, me semble-t-il, renforcer cette considération en faisant valoir que le toucher est une forme de perception par contact alors que la vue est une perception à distance. Par quoi j'entends que l'une des caractéristiques de la perception tactile est de supposer un contact direct avec l'objet perçu- où s'il est indirect, un contact faisant intervenir un intermédiaire rigide, comme, par exemple, la canne de l'aveugle —, alors que la perception visuelle suppose au contraire un objet placé à distance. Qu'il faille distinguer deux formes de perception semble en outre attesté par le fait que le sujet équipé du système de substitution visuo-tactile est capable dans une même situation de deux attitudes et peut avec un peu d'entraînement passer facilement de l'une à l'autre. Il peut soit se concentrer sur ce qui se passe dans son dos et il a alors une perception tactile des vibrateurs qui sont au contact de sa peau, soit se concentrer sur les objets balayés par la caméra dont il a une perception "visuelle".

Il reste encore possible d'objecter que les utilisateurs du SSVT ne voient pas à proprement parler parce qu'ils n'éprouvent pas de sensations proprement visuelles, par exemple, ils n'ont pas de sensations de couleurs. Comme le font voir les débats sur 
l'inversion du spectre colorél2 1 il faut ici distinguer deux choses. D'une part le fait que ces utilisateurs ne font pas les discriminations correspondant aux différences de couleur, d'autre part, le fait qu'ils n'ont pas les qualia éprouvés devant les différentes couleurs par les personnes dotées d'une vision normale. En ce qui concerne le premier point, il est vrai que pour le présent, le SSVT n'autorise pas la transmission des informations portant sur la longueur d'onde. Mais, comme le suggère Morgan (1977), il est en principe possible de concevoir un système qui, au lieu de ne comporter que des vibrateurs d'un même type, comporterait trois types de stimulateurs réagissant maximalement à des longueurs d'ondes différentes. Vraisemblablement, les utilisateurs de ce dispositif amélioré seraient capables de faire les mêmes discriminations entre couleurs que les sujets dotés d'une vision normale. En revanche, ils n'auraient pas de qualia de couleurs ou en tous cas n'associeraient pas les mêmes qualia que les sujets normaux à ces discriminations, s'il est vrai, comme le pensent la plupart des physiologistes, que ceux-ci sont liés à des événements dans des parties spécifiques du système nerveux. A supposer qu'ils en éprouvent, on peut spéculer qu'ils seraient plus semblables aux qualia tactiles habituels qu'aux qualia visuels. Ceci tendrait à suggérer qu'il peut y avoir dissociation entre le type de sensation éprouvé et le type de perception et par conséquent que des sensations d'un certain type ne sont pas des prérequis de la perception.

Notre question initiale — savoir si l'expérience rendue possible par le SSVT peut être considérée comme visuelle - dépend de la question de savoir si la vision se définit essentiellement comme expérience sensorielle ou comme expérience perceptive, ou en termes plus généraux des critères que nous utilisons pour distinguer les sens. Grice (1962) a proposé un inventaire de quatre critères. On peut distinguer les sens : (1) par rapport aux propriétés sur lesquelles ils nous informent (les odeurs pour l'odorat, les couleurs pour la vue, etc.); (2) par rapport aux caractéristiques subjectives des expériences éprouvées ; (3) par rapport aux différences des stimuli qui les activent; et (4) par référence aux organes impliqués.13

L'inconvénient principal du critère (1) dans sa formulation initiale est que certaines propriétés des objets peuvent être appréhendées par plusieurs sens 14 Par

12 Voir notamment, Block 1978; Hardin, 1988 ; Lycan, 1973 ; Shoemaker, 1975, 1982 ; van Gulick, 1993.

13 On trouvera des discussions des avantages et inconvénients de ces différents critères ainsi que de certains raffinements de ceux-ci notamment dans Roxbee-Cox (1970), Heil (1983) et Casati \& Dokic (1994).

14 Roxbee-Cox (1970) a proposé une modification de ce critère; il suggère que l'on considère non pas toutes les propriétés auxquelles donne accès un sens, mais que l'on associe à chaque modalité 
exemple, la forme et la texture peuvent jusqu'à un certain point être appréhendées tant visuellement que tactilement. D'après Heil (1983), le critère (2) se heurte à deux objections sérieuses : la première consiste à donner une caractérisation non-circulaire des qualia propres à chaque sens; la seconde à ne pas tomber dans l'anthropocentrisme. Intuitivement, nous voudrions pouvoir dire d'espèces très différentes qu'elles voient sans que cela nous oblige à supposer qu'elles ont les mêmes qualia visuels que nous. Le critère (4) ou critère des organes me semble pouvoir être ramené à l'un ou l'autre des trois autres critères. Etant donné la grande diversité anatomique et physiologique des organes sensoriels à travers les différentes espèces animales, si nous voulons éviter une inflation anarchique du nombre des sens, il faudra préciser selon quel critère on décide que deux organes sensoriels présentant des différences anatomiques et physiologiques qui peuvent être considérables - par exemple, l'œil humain et l'œil composé de l'abeille appartiennent néanmoins au même type —, il semble que, pour ce faire, nous devions donc en appeler à l'un des autres critères. Nous avons déjà vu que les critères (1) et (2) posaient divers problèmes. Reste à examiner le critère des stimuli. C'est le critère que défend Heil. La distinction entre les sens est fondée sur la différence entre les types de stimulations physiques exploités pour en tirer de l'information. D'après lui, par conséquent, "voir suppose une activité d'extraction d'information à partir des rayons lumineux ; il y a audition lorsqu'une créature extrait de l'information à partir d'ondes de compression de certains types ; l'odorat et le goût impliquent l'extraction d'information à partir de caractéristiques chimiques de l'environnement [...] ; le toucher fait intervenir la capacité à obtenir de l'information sur les choses par l'intermédiaire de certains types de contact mécanique" (1983 : 8). Ainsi que l'ont fait remarquer Casati et Dokic, ce critère n'est toutefois pas parfait en ce qu'il ne permet pas de distinguer entre perception visuelle et perception thermique, puisque dans les deux cas, le milieu semble être celui des radiations électro-magnétiques. L'objection n'est toutefois pas nécessairement dirimante, dans la mesure où, d'une part, radiations lumineuses et radiations thermiques se distinguent malgré tout par leur longueur d'onde et leur bande de fréquence. A défaut d'être parfait, le critère des stimuli semble donc moins défectueux que les autres critères

sensorielle une propriété-clé qu'elle seule permet de percevoir, par exemple la couleur pour la vision. Casati et Dokic (1994) soulignent toutefois que cette modification ne va pas sans poser de problèmes, car d'un côté “l'individualisation d'une propriété n'explique pas pourquoi nous effectuons une distinction entre voir une forme et la sentir au toucher; de l'autre, il nous faudrait admettre une multiplication démesurée des sens" (1994: 24), avec notamment autant de sens du toucher que de propriétés-clés distinctes. 
examinés. Il a en particulier l'avantage de mettre en évidence le caractère actif de la perception.

Le choix de ce critère, l'insistance sur le caractère actif de la perception, la mise en avant de la possibilité d'un divorce entre sensation et perception et par conséquent du caractère inessentiel de la sensation dans les processus perceptifs sont des thèmes qui font largement écho aux conceptions de Gibson. Gibson (1966) opère en effet une distinction entre l'information contenue dans une expérience et les aspects qualitatifs de ces expériences et, par suite, entre deux manières de concevoir les sens, soit comme canaux de sensations, essentiellement passifs, qui sont les sources des qualités conscientes de l'expérience, soit comme des systèmes perceptifs, essentiellement actifs, qui extraient ces informations et sont sources de connaissances sur sur le monde. Il souligne en outre l'absence de relation nécessaire entre sensation et perception: "la sensation n'est pas un prérequis de la perception, et les impressions sensorielles ne sont pas les 'données brutes' de la perception" (1966: 48); elles n'en sont qu'un accompagnement occasionnel. Certaines autres thèses de Gibson semblent également tout à fait compatibles avec les données fournies par le SSVT de Bach-y-Rita. C'est notamment le cas de sa thèse selon laquelle l'information 15 sur le monde est contenue dans les propriétés structurelles invariantes des stimuli. L'objet de la perception est donc d'extraire cette information en détectant ces invariants16 Dans ce processus de détection, le mouvement joue un rôle important. Les systèmes perceptifs sont susceptibles d'apprentissage, cet apprentissage ayant pour effet de permettre une

${ }^{15}$ La notion d'information, telle qu'elle est utilisée par Gibson présente de fortes similitudes avec la notion d'information de Dretske (1981, 1988). Ainsi, Gibson propose-t-il la caractérisation suivante: "information sur quelque chose signifie seulement spécificité par rapport à quelque chose. Par conséquent, quand nous disons que l'information est transportée par la lumière, ou par le son, l'odeur ou l'énergie mécanique, nous ne voulons pas dire que la source est litéralement transportée comme une copie ou une réplique. Le son de la cloche n'est pas la cloche et l'odeur du fromage n'est pas le fromage. Pareillement, la projection en perspective des faces d'un objet [...] n'est pas l'objet lui-même. Pourtant, dans tous ces cas une propriété du stimulus est univoquement reliée à une propriété de l'objet en vertu de lois physiques. C'est ce queje veux dire quand je parle de transport de l'information environnementale." (1966: 187)

${ }^{16}$ A insi Gibson écrit-il : “Par delà les changements dans les stimuli d'un lieu à l'autre et d'un temps à l'autre, on peut également montrer que certaines variables d'ordre supérieur - énergie du stimulus, rapports et proportions, par exemple - ne changent pas. Elles demeurent invariantes par rapport aux mouvements de l'observateur et aux changements d'intensité du stimulus. [...] Ces invariants du flux d'énergie qui arrive aux récepteurs d'un organisme correspondent aux propriétés permanentes de l'environnement. Elle constituent, par conséquent, de l'information sur l'environnement permanent." (1966 : 3) 
détection plus exacte, plus complète et plus rapide des invariants présents dans une stimulation.

Avant de passer à notre dernier problème, celui de la nature exacte du rôle du mouvement dans la perception spatiale, il est temps de faire un premier bilan et d'examiner jusqu'où la problématique à laquelle nous avons abouti à la suite du problème de Bach-y-Rita, se distingue de la problématique qui entourait à l'origine le problème de Molyneux et plus précisément de cette problématique telle que la concevait Berkeley. En premier lieu, on notera que Berkeley, d'un côté, Gibson et Bach-y-Rita de l'autre, s'accordent sur le fait que la fonction principale de la perception est de nous fournir des informations sur l'environnement. Il en découle que pour eux, le problème principal d'une théorie de la perception n'est pas d'expliquer pourquoi les choses ont l'apparence phénoménale qu'elles ont, mais de quelle manière les sens nous informent sur le monde. Le deuxième point commun important de ces auteurs est d'associer perception des propriétés objectives de l'environnement et invariance. Ainsi que nous l'avons vu, Berkeley lie la prééminence qu'il accorde aux idées tactiles par rapport aux idées visuelles au fait que les premières mais non les secondes sont constantes et Gibson rend compte de l'extraction d'information en termes de détection d'invariances.

A côté de ces similitudes, il faut noter deux différences capitales. Ni Berkeley, ni, à ma connaissance les auteurs du XVIIIle siècle, à l'exception peut-être de Thomas Reid (1785), ne songent à contester l'idée que la perception se fonde sur la sensation. La thèse berkeleyienne selon laquelle la spatialité est inhérente aux idées du toucher mais non à celles de la vue semble motivée par le fait qu'il existe une relation parfaitement univoque entre les sensations du toucher et les idées spatiales de grandeur, forme, distance, etc., ce qui suggère que les idées spatiales peuvent par conséquent être lues directement dans les sensations du toucher. En revanche, il n'y a pas de correspondance bi-univoque entre propriétés spatiales et sensations visuelles de couleur et d'illumination, d'où le caractère médiat de la perception visuelle spatiale. A l'opposé, Gibson insiste sur la séparabilité de la sensation et de la perception et sur l'indépendance de la perception vis-à-vis des sensations. Les expériences faites avec le SSVT de Bachy-Rita peuvent être tenues pour des démonstrations de cette indépendance. Pour Gibson, les fluctuations des sensations visuelles associées à la perception d'un objet à différentes distances ne donnent aucune raison de douter du caractère immédiatement spatial de la perception visuelle, puisque précisément la perception ne dépend pas de la sensation.

\section{Le rôle du mouvement dans la perception spatiale}


Posé en termes gibsoniens, le problème de la spatialité de la perception visuelle se décompose en deux questions : celle de la présence d'une information spatiale - sous forme d'invariants de structure - dans le stimulus visuel et celle de la capacité des organismes à extraire cette information. Les thèses les plus controversées de Gibson concernent la manière dont l'information est extraite par les organismes - la théorie de la saisie directe de l'information (direct pick-up of information). Nous n'avons pas le temps ici d'examiner ces thèses; il est néanmoins important de dire que l'on peut reconnaître à un organisme la capacité d'extraire les invariants présentés dans le stimulus sans souscrire sur ce point aux idées de Gibson17 En revanche, il est généralement reconnu que Gibson a démontré que l'information spatiale contenue dans les stimuli visuels était beaucoup plus riche qu'on ne l'avait pensé avant les travaux systématiques qu'il a menés 18 et qu'elle était ainsi suffisante pour donner une représentation non-ambiguë des propriétés spatiales de l'environnement. Si tel est le cas, il semblerait donc que Gibson ait raison contre Berkeley.

Toutefois, les démonstrations de Gibson reposent sur la substitution à la notion classique de stimulus visuel - conçu comme étant l'image formée par la lumière sur la rétine à un instant donné - d'une notion plus riche, celle de la rangée optique (optic array) et de ses transformations, que nous allons examiner. Cette redéfinition de la notion de stimulus visuel a pour conséquence d'inclure le mouvement dans le stimulus puisque l'une des sources principales des transformations de la rangée optique sera le mouvement du spectateur. La question de la spatialité intrinsèque à la perception visuelle ne recevra donc une réponse positive que pour autant qu'en élargissant ainsi la notion de stimulus visuel, on ne se donne pas subrepticement des idées spatiales déjà constituées. D'où la nécessité d'élucider le rôle exact joué par le mouvement dans la perception visuelle.

Commençons par préciser le rôle attribué au mouvement par Gibson. D’abord qu'entend-il exactement par rangée optique? Elle est définie comme la projection sphérique d'une configuration géométrique de lumière ambiante autour d'un point d'observation. La rangée optique existe donc objectivement indépendamment de l'existence d'un observateur. Elle constitue un stimulus potentiel qui devient actuel

17 Selon Gibson, le système visuel est constitué de telle sorte qu'il enregistre automatiquement certaines dimensions d'invariance dans le flux de stimulation : “il entre en résonance avec la structure invariate ou encore est à l'unisson de celleci"(Gibson, 1986 : 249). A l'opposé, des théoriciens de la vision comme Marr soutiennent que l'extraction des invariants est une tâche complexe de traitement de I'information dont Gibson a sérieusement sous-estimé la difficulté (Marr, 1982 : 30).

18 Pour une présentation générale de ces travaux, voir Gibson (1950). 
lorsqu'un œil se trouve au point d'observation. Toutefois, on ne doit pas identifier stimulus actuel et stimulus potentiel à un instant donné, car un stimulus actuel est étendu dans le temps : à un stimulus actuel peut donc correspondre une série de transformations de stimuli potentiels, qui peut être soit l'effet d'un déplacement de l'œil (mouvement de l'observateur), soit l'effet d'un changement dans l'environnement. Un stimulus actuel possède donc à la fois une structure simultanée - la configuration de la rangée optique à un instant donné - et une structure successive donnée par les transformations de la rangée optique. Pour Gibson, si l'information spatiale contenue dans un stimulus est en partie donnée dans ses invariants statiques, elle l'est néanmoins surtout par ses invariants dynamiques. L'importance du mouvement tient donc en tout premier lieu à son rôle dans la production de ces invariants.

Ceci ne fait pas de Gibson un défenseur de la théorie motrice de la perception spatiale, au sens où une telle théorie fait consister l'apprentissage de la perception spatiale visuelle en la mise en place de corrélations entre stimuli visuels et idées motrices, telles qu'en présence de tels ou tels types d'objet dans son champ visuel, le sujet déclenche un programme moteur adapté. Gibson prend d'ailleurs soin de distinguer entre deux types de mouvements : d'une part, les mouvements exploratoires dont l'objet est d'obtenir des stimulations de l'environnement en vue de la perception et, d'autre part, les mouvements performatifs qui servent à la réalisation des comportements au sens ordinaire du terme.

Toutefois, il faut encore demander, premièrement, si l'utilité du mouvement dans la détection des invariants spatiaux n'a pas pour condition préalable la capacité de distinguer, d'une part, les transformations de la rangée optique qui déroulent des mouvements de l'observateur des transformations liées à des changements dans l'environnement et, d'autre part, les déplacements simplement passifs de l'observateur de ses mouvements auto-générés. Et il faut demander, deuxièmement, si la possession d'une telle capacité n'implique pas que l'observateur dispose déjà de représentations spatiales. On peut, semble-t-il, reprocher à Gibson de n'avoir pas bien perçu la différence des rôles joués par les déplacements passifs et les déplacements actifs. Il insiste sur la nécessité du mouvement pour la mise en évidence de certains des invariants structurels du stimulus visuel, mais ne semble pas appréhender toute l'importance du mouvement spontané pour la localisation dans le monde extérieur des invariants identifiés. En revanche, ainsi que nous l'avons vu, Bach-y-Rita et ses collègues sont très sensibles au rôle important joué par le mouvement actif 19 Ils

19 Ce rôle est également mis en évidence dans les importants travaux de Held sur le rôle du mouvement dans la perception (Held, 1965; Held \& Bauer, 1967, Held \& Hein, 1963). 
avancent l'hypothèse qu' "une corrélation précise de la translation de l'entrée avec le mouvement auto-généré du senseur est la condition nécessaire et suffisante d'une attribution des phénomènes observés à un monde extérieur stable" et que "l'hypothèse converse de cette hypothèse devrait également être vraie - à savoir qu'un défaut de correspondance entre la translation de la rangée d'input et les mouvements auto-générés du senseur devraient résulter en des expériences attribuées soit à des conditions nonrigides de l'environnement externe, soit à des phénomènes dont l'origine perçue est interne à l'observateur “(White et al., 1970 : 25). Il semble qu'une petite correction de cette hypothèse s'impose : il est plausible que l'existence d'une corrélation précise entre les transformations de l'input et les mouvements auto-générés soit une condition nécessaire, mais cette condition n'est pas suffisante, car ce qui est crucial ce n'est pas simplement que la corrélation existe, mais encore qu'elle soit détectée comme telle. En termes gibsoniens, cette corrélation peut être tenue pour un invariant d'ordre supérieur et l'hypothèse de Bach-y-Rita serait ou devrait être que la perception du monde externe comme tel dépend de la détection de cet invariant. La conception du rôle du mouvement que je viens d'esquisser succintement semble présenter de fortes affinités avec la théorie qu'a développée Poincaré du rôle du mouvement dans la représentation de l'espace. Aussi le meilleur moyen de préciser cette conception sera-t-il de le faire par référence à la théorie de Poincaré.

Poincaré $(1905,1907,1930)$ opère une distinction entre l'espace géométrique et l'espace représentatif. Les caractéristiques essentielles de l'espace géométrique sont d'être continu, infini, tridimensionnel, homogène et isotrope20 L'espace représentationnel constitue une approximation sensible de l'espace géométrique. Le problème de Poincaré est d'expliquer comment nous sommes capables de construire cet espace représentationnel qui approche l'espace géométrique sur la base de nos espaces sensoriels, l'espace visuel, l'espace tactile et l'espace moteur, étant donné que leurs caractéristiques sont très éloignées des caractéristiques de l'espace géométrique. L'espace visuel pur est bidimensionnel, il est limité, il n'est pas homogène. La troisième dimension a sa source dans des sensations musculaires - les sensations de convergence et d'accommodation — très différentes de sensations visuelles. L'espace visuel complet, tridimensionnel, n'est donc pas non plus isotrope. Les espaces tactiles et moteurs s'éloignent encore plus de l'espace géométrique, l'espace moteur ayant d'une certaine manière autant de dimensions qu'il y a de muscles.

20 Un espace est homogène si tous ses points sont identiques entre eux ; il est isotrope si toutes les droites qui passent par un même point sont identiques entre elles. 
La réponse de Poincaré à ce problème consiste à considérer qu'il ne faut pas chercher l'origine de l'espace représentatif quasi-géométrique dans nos sensations prises isolément mais dans les lois suivant lesquelles ces sensations se succèdent. La construction de l'espace trouve son origine dans la possibilité de distinguer deux types de changements de nos impressions. Le passage d'un ensemble d'impressions A à un ensemble d'impressions B correspond à un changement de position de l'objet qui cause ces impressions, s'il est possible de restaurer l'ensemble d'impressions A en faisant des mouvements qui nous replacent vis-à-vis de l'objet dans la situation relative initiale. Il y a donc changement de position lorsqu'il est possible de corriger une modification qui s'est produite et de rétablir l'état initial par une modification inverse. Il y a changement d'état lorsqu'une telle correction n'est pas possible. Par essais et erreurs, nous apprenons quels mouvements volontaires sont susceptibles de compenser quels changements. C'est parce que cette association par compensation exprime sensiblement une loi de groupe, le groupe des mouvements rigides, que l'espace représentatif ainsi acquis constitue une approximation de l'espace géométrique.

Est-ce à dire qu'en apprenant de l'expérience quand des changements d'impressions sensorielles sont susceptibles d'être compensés par des mouvements volontaires et par quels mouvements, nous apprenons en fait à transférer aux représentations de la vue ou du toucher des notions spatiales déjà présentes dans la représentation des mouvements? Poincaré refuse une telle interprétation. Par représentation des mouvements, il n'entend pas la représentation des mouvements dans l'espace, mais la représentation des sensations musculaires qui les accompagnent et qui en elles-mêmes ne présupposent pas la notion d'espace.

On notera que, même si Poincaré adopte ici un point de vue périphéraliste en parlant de sensations musculaires, la désaffection actuelle pour l'approche périphéraliste ne menace pas directement sa position. Le débat entre périphéralisme ou théorie de la réafférence et centralisme ou théorie de l'efférence porte sur la manière dont nous sommes informés de nos propres productions motrices 21 Selon les centralistes, le système nerveux s'informe lui-même de ses propres productions motrices : lorsque le cortex moteur envoie une commande motrice en direction des muscles, il produit simultanément à l'attention d'autres parties du cortex une copie d'efférence ou décharge corollaire qui les informe de cette commande. Selon les périphéralistes, en revanche, nous sommes informés de nos productions motrices par le feedback proprioceptif (réafférences) lors de l'exécution de la commande. Mais la réponse à la question qui

${ }^{21}$ Pour un historique de ce débat et un plaidoyer en faveur de la théorie centraliste, cf. Jeannerod, 1983. 
nous intéresse ne semble pas dépendre directement de l'issue de cette controverse entre centralistes et périphéralistes. Que les représentations de sa propre activité motrice dont dispose un organisme soient d'origine périphérique ou centrale ne nous importe dans le cadre présent que pour autant que l'un ou l'autre type de représentations doive ou non nécessairement avoir un caractère spatial. Or, quoi qu'il en soit empiriquement, sur un plan strictement conceptuel, il ne semble pas que les représentations périphériques ou que les représentations centrales doivent nécessairement avoir un contenu spatial. Ce qui est nécessaire, si l'on en tient pour le centralisme, c'est qu'à des commandes motrices aboutissant à des mouvements différents soient associées des représentations différentes ; ce qui importe, si l'on se veut périphéraliste, c'est qu'à des mouvements différents soient associées des sensations proprioceptives différentes. La seule chose qui importe sur le plan conceptuel c'est l'existence d'une corrélation précise entre mouvements et représentations motrices, telle qu'à des mouvements différents soient associées des représentations motrices différentes. Mais rien ne semble exiger que ce jeu de différences s'exprime en termes spatiaux.

En résumé, selon Poincaré, l'espace ne nous est donné ni dans nos représentations sensorielles, ni dans nos représentations motrices. L'espace est toutefois construit sur la base de ces représentations. Cette construction suppose (1) que soient considérées non simplement des ensembles de sensations simultanées, mais des suites de sensations, (2) que soient considérées à la fois des suites de sensations externes (visuelles, tactiles, etc.) et des suites de sensations de mouvement et (3) qu'une association par compensation mette en corrélation des suites de sensations extéroceptives avec des suites de sensations musculaires. L'association par compensation permet ainsi la construction ou l'extraction des invariants du groupe des mouvements rigides. En réalisant un modèle sensible de ce groupe, l'association construit un espace représentatif qui constitue une approximation sensible de l'espace géométrique. On notera, à la suite de Vuillemin⒉ que l'association par compensation doit être distinguée de l'association des empiristes classiques. A la différence de l'association empiriste, l'association par compensation est un processus actif : c'est l'opération d'une organisation sensible active — la motricité volontaire sur des suites de sensations passives qui permet la classification de ceux-ci et la construction d'invariants spatiaux. L'espace représentatif n'est ni donné par les sens ni inné, il est activement construit. Le seul élément inné nécessaire à cette construction réside, selon Poincaré, dans l'idée de groupe de transformations ; la condition interne nécessaire à la mise en œuvre de cette idée est la capacité de se mouvoir et la condition

22 “L'espace représentatif selon Poincaré", conférence donnée à l'occasion du Colloque International Henri Poincaré, Nancy 14-18 mai 1995. 
externe pour la construction d'une géométrie sensible est l'existence dans la nature de corps solides.

La théorie de Poincaré semble donc permettre de donner un contenu précis à ce qui est évoqué de manière vague lorsque l'on parle de processus actifs d'extraction d'invariants et que l'on dit que nos représentations spatiales sont le produit de tels processus. Mais, inversement, il est peut-être possible de mieux éclairer, voire de corriger certains aspects, de la théorie de Poincaré en faisant intervenir certaines distinctions formulées notamment par Gibson. Poincaré utilise le terme d'espace représentatif à la fois pour désigner l'approximation sensible de l'espace géométrique qui est le produit de l'association par compensation et pour désigner les espaces visuel, tactile ou moteur dont il souligne l'hétérogénéité et l'éloignement vis-à-vis de l'espace géométrique. Le double usage ainsi fait du terme d'espace représentatif recèle, me semble-t-il, une ambiguité. Ainsi que nous l'avons vu, suivant Gibson, nous devons distinguer l'information contenue dans une expérience sensorielle de la qualité de cette expérience sensorielle. Alors que la sensation correspond à la qualité de l'expérience, la perception dépend de l'exploitation de l'information contenue dans l'expérience. Quand Poincaré parle de l'espace visuel, il peut en principe faire référence soit au type de structure spatiale de l'information contenue dans le stimulus visuel statique (la structure spatiale de l'image rétinienne), soit à l'espace de la sensation visuelle (le champ visuel), soit à l'espace visuellement perçu (le monde visuel). La caractérisation qu'il donne de l'espace visuel laisse à penser qu'il oscille entre la première et la seconde possibilité, alors qu'une utilisation univoque du terme d'espace représentatif supposerait qu'il fasse référence à l'espace visuellement perçu. Cette équivoque dans l'usage de l'expression d'espace représentationnel amène Poincaré à soutenir que les représentations de l'espace issues des différentes modalités perceptives sont hétérogènes les unes par rapport aux autres. Mais si l'on applique les distinctions gibsoniennes, on peut donner une autre interprétation de la théorie de Poincaré. Ce que montre alors la description par Poincaré des espaces visuel, tactile et moteur, c'est l'hétérogénéité des modes de structuration de l'information spatiale dans les stimuli associés aux différentes modalités, plus précisément c'est l'hétérogénéité de la structuration de l'information dans les ensembles de sensations simultanées des différentes modalités. En revanche, le mode de structuration dynamique des stimuli est le même quelle que soit la modalité concernée ; c'est ce qui rend possible l'association par compensation et ce qui explique pourquoi l'espace visuel et l'espace tactile sont un seul et même espace. Poincaré nie donc d'une certaine manière l'existence de sensibles communs dans la mesure où il soutient que le format selon lequel l'information spatiale est codée dans la structure simultanée des impressions sensorielles varie d'une modalité à l'autre. Mais sa théorie affirme en 
revanche l'existence de "perceptibles communs", dans la mesure où l'information spatiale contenue dans la structure dynamique des stimuli peut être ressaisie par le même processus d'association par compensation, quelle que soit la modalité sensorielle considérée.

Même si l'on veut bien concéder que cette double relecture de Bach-y-Rita et Gibson par Poincaré et de Poincaré par Bach-y-Rita et Gibson n'est pas totalement extravagante, on pourra objecter que cette reconstruction montre certes qu'il est en principe possible de construire des représentations spatiales objectives sur la base d'informations fournies par une modalité perceptive ainsi que d'informations sur notre propre activité motrice, mais qu'elle ne montre pas que cela soit effectivement la manière dont nous construisons nos représentations spatiales. En d'autres termes, Poincaré montre que, pour rendre compte de l'existence de représentations spatiales objectives, il n'est pas en principe nécessaire de postuler l'existence d'une notion d'espace préexistant à toute expérience; il ne montre pas que nous ne disposons pas en fait d'une telle notion.

On peut, semble-t-il, admettre que l'association par compensation soit à la base de notre représentation de l'espace tout en niant que nous devions apprendre par essais et erreurs quels mouvements volontaires compensent quelles modifications des sensations. L'objection peut prendre au moins deux formes. On peut mettre l'accent sur l'existence de mécanismes automatiques innés de compensation. Un certain nombre d'études menées par les psychologues sur les bébés ont mis en évidence l'existence de diverses formes de corrélations intermodales préalablement à tout apprentissage. Par exemple, la découverte par Meltzoff (Meltzoff \& Moore, 1983, 1989; Meltzhoff, 199à) de la capacité de très jeunes bébés à imiter des expressions faciales vues suggère l'existence de correspondances innées entre système perceptif visuel et système moteur. Plus simplement, la capacité à suivre du regard la trajectoire d'un objet semble témoigner de l'existence de mécanismes innés et automatiques de compensation. A-t-on là affaire à des contre-exemples suffisant à saper la thèse de Poincaré ? Vraisemblablement non. L'existence de mécanismes automatiques de compensation ne suffit pas à garantir l'existence d'une représentation objective de l'espace. L'articulation observée entre, par exemple, stimuli visuels et comportements moteurs n'exige pas la médiation d'un niveau intermédiaire de représentation spatiale. Les stimuli visuels peuvent être automatiquement corrélés à des comportements moteurs sans qu'il soit nécessaire d'en construire préalablement une interprétation distale spatiale. Tout au plus peut-on dire que l'information spatiale est représentée sous formes de procédures, mais l'information ainsi représentée n'est pas exploitable pour d'autres opérations du système cognitif, elle ne fait pas l'objet d'une représentation détachée. La théorie de Poincaré insiste en 
revanche sur le fait que l'espace représentatif suppose la représentation de la compensation, suppose l'opération de mouvements volontaires qui font l'objet d'une représentation consciente. En bref, ce qui est en jeu ce n'est pas la capacité à se comporter de manière spatialement appropriée, c'est la capacité de se représenter l'espace. Ce que l'existence de mécanismes automatiques de compensation suggère, c'est que nous avons une capacité innée à nous comporter de manière spatialement appropriée, mais non que nous avons une notion innée d'espace.

La seconde forme que peut prendre l'objection est plus directe. Partant de l'axiome qu'il n'y a rien d'incohérent à supposer que tout ce qui est appris ou peut être appris pourrait également être inné, 23 elle demande quelles raisons on a, dans le cas de l'espace, de soutenir que les concepts spatiaux doivent être acquis plutôt qu'innés. Pourquoi penser que chacun devrait en passer par le processus tâtonnant de l'apprentissage pour construire l'espace représentatif, plutôt que supposer que l'évolution, en bonne marraine qu'elle est, nous a gracieusement doté de concepts spatiaux tout faits, de telle sorte que nos sensations visuelles ou tactiles donnent immédiatement lieu à des représentations spatiales objectives ? Dans le cas de l'espèce humaine, deux types de considérations me semblent faire pencher la balance en faveur de l'acquisition plutôt que de l'innéité. Il s'agit en premier lieu des résultats des expériences de mise en conflit des modalités sensorielles, comme par exemple le décalage de l'information visuelle qu'induit le port de prismes. Chez l'espèce humaine et chez le singe, à la différence d'autres espèces moins évoluées, l'adaptation est rapide et une nouvelle corrdination s'établit bientôt. Mais les expériences de Held24 ont en outre montré que cette recoordination ne se produit, d'une part, que si la déformation de la relation entre les informations livrées par les modalités en conflit est systématique et constante et, d'autre part, que si le sujet exécute des mouvements de manière active dans son environnement. On peut voir dans ces deux contraintes la transposition de deux conditions qui, selon Poincaré, doivent être satisfaites pour que la construction d'un espace représentationnel quasi-géométrique soit possible (un être capable de se mouvoir volontairement et un monde comportant des corps solides). En particulier la contrainte de déformation systématique et constante est nécessaire si l'on veut préserver la distinction entre changements d'état et changements de position rendue possible par l'existence de corps rigides.

23 On retrouve cet "axiome" dans la discussion par Evans (1985 : 378) du problème de Molyneux.

24 Pour les références des travaux de Held, cf. note 16. Pour une étude de l'adaptation perceptive et des modes de coordination possibles des modalités perceptives, cf. Rock (1966). 
En quoi les résultats de ce type d'expériences tendent-ils à corroborer la théorie de Poincaré plutôt qu'une hypothèse innéiste rivale ? Si l'on adopte la théorie de Poincaré, la possibilité de la recoordination et la nécessité de mouvements actifs pour que celle-ci ait lieu s'expliquent sans que l'on doive faire appel à des hypothèses supplémentaires. Dans une optique innéiste, où pour chaque modalité des mécanismes innés assurent le passage des informations sensorielles à des représentations spatiales, on ne peut expliquer la résolution des conflits entre modalités et l'harmonisation des représentations spatiales qui en sont issues sans faire l'hypothèse de mécanismes supplémentaires en charge d'arbitrer ces conflits. En outre, il existe en principe plusieurs manières possibles de mettre en conflit les modalités et pour chaque conflit créé plusieurs manières possibles de recoordonner les modalités. A supposer que les mécanismes d'arbitrage tendent à recoordonner les modalités de manière à préserver la véridicité des représentations spatiales, on ne voit guère comment ils y parviendraient sans prendre en compte les informations délivrées par le mouvement volontaire ou plus précisément sans exploiter un principe similaire à celui de l'association par compensation. En bref, en choisissant la voie innéiste, on ne fait que reculer pour mieux sauter, puisque les hypothèses sur le rôle du mouvement volontaire, écartées à un premier niveau, doivent être réintroduites pour rendre compte de la possibilité de la recoordination.

L'hypothèse innéiste n'est pas pour cela contradictoire, mais une explication "à la Poincaré" a sur elle l'avantage de la simplicité. Encore une fois, on pourra objecter que cet avantage n'est pas décisif. Mais on peut encore faire intervenir un deuxième type de considérations en faveur d'une hypothèse à la Poincaré. Ces considérations sont tirées de l'expérience Bach-y-Rita elle-même. Reprenons l'hypothèse innéiste sous un autre angle. Selon cette hypothèse, il existe des mécanismes innés qui assurent le passage des informations spatiales dont sont porteuses les modalités à une représentation spatiale objective. Dans la mesure où le mode de codage de l'information spatiale diffère selon les modalité 25 on doit supposer que chacun de ces mécanismes a son mode d'opération propre adapté à la modalité à laquelle il est dédié. Or que se passe-t-il chez les sujets équipés du SSVT ? Ceux-ci reçoivent via la matrice de vibrateurs placée sur leur peau des informations spatiales qui ont été véhiculées par des signaux lumineux et dont le mode de structuration est (approximativement) de type visuel. Or les voies cérébrales qui interviennent dans le traitement de ces stimuli procurés par le SSVT ne sont pas les voies cérébrales qui interviennent normalement dans le traitement des stimuli visuels,

25 On notera que cette différence est le résultat de la différence conjuguée de la structure des signaux et de celle des récepteurs selon les modal ités. 
mais celles qui interviennent dans le traitement des stimuli tactiles. Si l'on s'en tient à l'hypothèse innéiste, on n'est pas en mesure d'expliquer comment le traitement de ces stimuli peut donner lieu à une représentation spatiale constituant une interprétation correcte de l'information délivrée. Si l'hypothèse innéiste était juste, les stimuli ne devraient pas pouvoir être interprétés autrement que comme des configurations tactiles, des "dessins" sur la peau. En revanche, les résultats de Bach-y-Rita s'expliquent facilement dans une hypothèse à la Poincaré, puisque dans cette optique, quelle que soit la modalité considérée, c'est le même processus d'association par compensation qui permet la construction d'une représentation spatiale. Que les stimuli visuels présentés tactilement ne donnent pas lieu aux mêmes représentations spatiales que les stimuli tactiles ordinaires tient à ce que l'association par compensation ne dégage pas les mêmes invariants dans les deux cas.

Les travaux des psychologues, l'étude de dispositifs tels que le SSVT, mettent en évidence le caractère actif de la perception et l'importance du rôle du mouvement volontaire dans la constitution de représentations spatiales objectives. J'ai essayé de montrer que la théorie de Poincaré donnait une explication cohérente de ces phénomènes. J'ai également essayé de montrer que cette théorie qui affirme le caractère acquis de l'espace représentatif quasi-géométrique était susceptible de rendre compte plus facilement des phénomènes d'adaptation perceptive et des résultats de l'utilisation du SSVT que sa rivale innéiste. A la fin de son Essai, Berkeley avait imaginé une pure intelligence dotée simplement du sens de la vue et il niait qu'elle puisse avoir des représentations spatiales. Nous pouvons résumer notre propos en disant que, si nous reprenions l'expérience de pensée de l'esprit désincarné de Berkeley, mais qu'avec à peine plus de générosité nous attribuions à cette intelligence, outre la vue, un œil mobile, des représentations motrices correspondantes et l'idée de groupe de transformation, il faudrait conclure, contre l'opinion de Berkeley, qu'elle aurait des représentations spatiales et que, de surcroît, sa représentation de l'espace serait une approximation grossière de l'espace géométrique

Je voudrais préciser un dernier point avant de conclure. Imaginons que la créature de Berkeley se voie maintenant dotée d'un deuxième sens, le tact par exemple, et que, par le jeu de l'association par compensation, elle acquière des représentations spatiales tactiles. Étant donné son mode de construction, l'espace tactile ainsi obtenu sera, comme l'espace visuel, une approximation d'un espace géométrique, continu, infini, tridimensionnel, homogène et isotrope. Mais ces deux espaces visuel et tactile fusionneront-ils en un espace unique ou se combineront-ils pour former un espace à six dimensions ? Il me semble que la condition nécessaire à leur fusion est que les mêmes suites de mouvements qui fonctionnent comme des opérations inverses relativement aux 
suites de sensations tactiles puissent également fonctionner comme opérations inverses pour les suites de sensations visuelles qui se déroulent simultanément. Dans l'exposé de sa théorie, Poincaré impose comme restriction la seule considération des mouvements volontaires qui induisent un déplacement en bloc du corps, sans déformation de celui-ci. Cette restriction nous donne la condition nécessaire de la fusion. Supposons, au contraire, que la créature de Berkeley, décidément monstrueuse, soit dotée à la fois d'une main mobile et d'un œil mobile, dont les mouvements soient totalement indépendants, qu'elle ne puisse pas se mouvoir en bloc et, pour plus de précautions encore, qu'elle ne puisse voir sa main ou toucher son œil. Une telle créature pourrait par hypothèse avoir des représentations spatiales visuelles et des représentations spatiales tactiles, mais elle n'aurait aucun moyen de corréler ces espaces et de les réduire l'un à l'autre. 26 L'humain ordinaire, dont certains déplacements mais non tous sont rigides, constitue un cas intermédiaire. Pour qu'il puisse intégrer ses différents espaces perceptifs, plusieurs conditions sont requises. Cette intégration suppose, en premier lieu, que soit remplie une condition théorique de commensurabilité. Or cette première condition peut en principe être remplie, précisément parce que les espaces perceptifs, étant tous le résultat d'une association par compensation, ont en commun d'être des approximations de l'espace géométrique tridimensionnel. Nous remplissons également une deuxième condition nécessaire: nous pouvons nous servir simultanément de plusieurs sens. Enfin, nous sommes capables de certains déplacements rigides ainsi que de déplacements qui ne modifient pas la position relative de différents récepteurs sensoriels. Nous sommes donc en mesure d'apprendre de l'expérience que certains déplacements constituent des opérations inverses pour des suites d'impressions dans plusieurs modalités. Tous nos déplacements n'ont toutefois pas cet effet. Si je ne bouge que les yeux, je n'induis des modifications que dans mes sensations visuelles. Une intégration complète de nos espaces perceptibles devra donc aller de pair avec la construction d'une représentation spatiale du corps propre. Les discordances entre nos espaces perceptifs ne peuvent disparaître que si nous nous représentons nous mêmes comme occupants de l'espace et comme susceptibles de certaines déformations spatiales. Il importe de souligner ici qu'insister sur le fait qu'une représentation objective une et cohérente de l'espace exige que nous disposions d'une représentation spatiale du corps propre ne revient pas à introduire en contrebande une spatialité déjà constituée. Nos sensations proprioceptives n'ont pas plus que nos sensations visuelles ou tactiles à être d'emblée spatiales et le même processus d'association par

${ }^{26}$ Cette question de la commensurabilité d'espaces appréhendés par des modalités différentes est le thèmed'un article de Eilan (1993b). 
compensation peut en principe expliquer la genèse d'une représentation spatiale du corps propre. Par conséquent, la représentation spatiale du corps propre n'a pas de statut d'exception ni de privilège épistémique particulier, mais son existence est nécessaire à l'intégration cohérente des différents espaces perceptifs.

\section{Conclusion}

Si l'on admet que ce qui est essentiel dans le problème de Molyneux n'est pas le débat entre innéisme et empirisme, mais la question de savoir si la perception spatiale est modale et, corrélativement, si la perception visuelle est ou non intrinsèquement spatiale, l'une des grandes différences entre l'espace conceptuel dans lequel s'inscrivait la problématique au XVIIIème siècle et l'espace contemporain consiste en une modification radicale de la conception des rapports entre perception et sensation. L'expérience de Bach-y-Rita illustre la possibilité d'une indépendance des sensations et des perceptions visuelles. Nos perceptions visuelles n'ont pas à se fonder sur des sensations visuelles. Ce divorce des sensations et des perceptions rend caduque l'une des principales stratégies argumentatives du XVIIIème siècle : qu'il n'y ait rien de commun entre sensations visuelles et sensations tactiles n'implique pas que les idées spatiales attachées aux unes ne puissent l'être aux autres. En termes contemporains, le caractère modal des sensations n'implique nullement, par lui-même, le caractère modal des perceptions. Le débat contemporain se distingue encore du débat dix-huitièmiste par une insistance beaucoup plus nette sur le caractère actif de la perception, par opposition au caractère passif de la sensation. Au regard de cette redéfinition des rapports entre perception et sensation et des résultats des expériences de Bach-y-Rita, si l'on s'intéresse aux sens en tant que systèmes perceptifs et non pas en tant que canaux des sensations, alors le meilleur critère de classification de ceux-ci est sans doute le critère des stimuli. En outre, quoique tant Berkeley que des théoriciens contemporains comme Gibson et Bach-y-Rita insistent sur le rôle important du mouvement dans la perception de l'espace, cette insistance sur le rôle du mouvement est associée à des conclusions opposées quant au caractère intrinsèquement spatial de la perception visuelle. J'ai essayé de montrer que la théorie de la construction de l'espace représentatif de Poincaré permettait de rendre compte de l'importance du mouvement volontaire dans la perception et de donner un contenu précis à la notion d'extraction d'invariants utilisée par les psychologues de la perception. Dans cette optique, insister sur le rôle du mouvement dans la perception visuelle ne revient donc pas à introduire frauduleusement une notion d'espace préalable à toute expérience. Inversement, j'ai essayé de montrer 
que si l'on relit la théorie de Poincaré à la lumière des distinctions contemporaines entre qualités de l'expérience et information contenue dans celle-ci, entre sensation et perception, cette théorie peut être réinterprétée, d'une part, comme une théorie dynamique de la perception spatiale dans la mesure où elle met l'accent sur la structuration dynamique des stimuli et, d'autre part, relativement à la question de l'espace, comme une théorie des "perceptibles communs". Si les invariants spatiaux sont principalement détectables dans la structure dynamique des stimuli et si la structuration dynamique des stimuli est la même pour toutes les modalités, nos représentations spatiales perceptives en tant qu'elle résultent de la détection de ces invariants dynamiques sont en principe compatibles entre elles. Le problème de Bach-y-Rita, qui m'a inspiré cette double réinterprétation de Gibson par Poincaré et de Poincaré par Gibson, et son illustre ancêtre, le problème de Molyneux, apparaissent alors susceptibles de recevoir une solution positive. 


\section{BIBLIOGRAPHIE}

Bach-y-Rita, P., 1972, Brain Mechanisms in Sensory Substitution, New-York : Academic Press.

Bach-Y-Rita, P. , Collins, C. C., W., Saunders, F.A., White, B, \& L. .Scadden, 1969, "Visual substitution by tactile image projection", Nature, 221, pp. 963-964.

Berkeley, G., 1709, Essai pour une nouvelle théorie de la vision, trad. collective sous la direction de G. Brykman, Euvres, t. I, Paris, PUF, 1985.

Block, N., 1978, "Troubles with functionalism", dans w. Savage (ée.) Perception and Cognition: Minnesota Studies in the philosophy of Science, vol. IX, Minneapolis, University of Minnesota Press.

Bower, T. G. R., 1982, Development in Infancy, seconde édition révisée, San Francisco : W. H. Freeman \& Company.

Casati, R. \& Dokic, J, 1994, La philosophie du son, Paris : Éditions Jacqueline Chambon.

Cassirer, E., 1932, La philosophie des Lumières, traduction fr. de P. Quillet, Paris : Fayard, 1966.

Collins, C. C. \& P. Bach-y-Rita, 1973, "Transmission of pictorial information through the skin", Advances in Biological and Medical Physics, 14, pp. 285-315.

Condillac, 1754, Traité des sensations, "Corpus des œuvres de philosophie en langue française, Paris : Fayard, 1984.

Dennett, D., 1988, "Quining Qualia”, in A. J. Marcel \& E. Bisiach (eds) Consciousness in Contemporary Science, Oxford : The Clarendon Press, pp. 42-77.

Diderot, D., 1749, Lettre sur les aveugles, dans CEuvres, Paris, Pléiade, Gallimard, 1951, pp. 811-861.

Dretske, F., 1981, Knowledge and the Flow of Information, Cambridge, Mass. : MIT Press.

Dretske, F., 1988, Explaining Behaviour, Cambridge, Mass. : MIT Press.

Eilan, N., 1993a, "Introduction: Spatial in the sensory modalities", dans Eilan, McCarthy \& Brewer (éds), pp. 179-190.

Eilan, N., 1993b, "Molyneux's question and the idea of an external world"”, dans Eilan, McCarthy \& Brewer (éds), pp. 236-255.

Eilan, N., McCarthy, R. \& Brewer, B., (éds), 1993, Spatial representation, , Cambridge, Mass. : Blackwell.

Evans, G.L, 1985, "Molyneux question”, dans Collected Papers. Oxford : Clarendon Press, pp. 364-399.

Gibson, J. J., 1950, The perception of the visual world, Boston : Houghton Mifflin Company.

Gibson, J. J., 1966, The senses considered as perceptual systems, Boston : Houghton Mifflin Company.

Grice, H. P. , "Some remarks about the senses", dans R. J. Butler (éd.), Analytical Philosophy, Oxford : Basic Blackwell.

Hardin, C. L., 1986, Color for Philosophers, Indianapolis, IN : Hackett.

Hatfield, G., 1990, The natural and the normative, Cambridge, Mass. : Bradford Books, MIT Press.

Heil, J., 1983, Perception and cognition, Berkeley : University of California Press.

Held, R., 1965, "Plasticity in sensory-motor systems", Scientific American, 213, pp. 84-94.

Held, R. \& J. A. Bauer, 1967, "Visually guided reaching in infant monkeys after restricted rearing, Science, 155, pp. 718-720.

Held, R. \& A. Hein, 1963, Movement-produced stimulation in the development of visually guided behavior", Journal of comparative and physiological psychology, 56, pp. 872-876.

Jeannerod, M., 1975, "Déficit visuel persistant chez les aveugles-nés opérés", Année Psychologique, 75, pp. 169-196.

Jeannerod, M., 1983, Le cerveau-machine, Paris, Fayard.

Leibniz, G. W., 1765, Nouveaux essais sur l'entendement humain, Paris, Garnier-Flammarion, 1966.

Lycan, W., 1973, "Inverted Spectrum”, Ratio XV, 315-19.

Meltzhoff, A. N., 1990, "Towards a developmental cognitive science : The implications of cross-modal matching and imitation for the development of representation and memory in infancy", dans A. Diamond (éd.), The Development and Neural Basis of Higher Cognitive Functions. Annals of the New-York Academy of Sciences, 608, pp. 1-31.

Meltzhoff, A. N. \& Moore, M. K., 1983, "Newborn infants imitate adult facial gestures", Child Development, 54, pp. 702-9.

Meltzhoff, A. N. \& Moore, M. K., 1989, "Imitation in newborn infants : Exploring the range of gestures imitated and the underlying mechanisms", Developmental Psychology, 25, pp. 954-62.

Mérian, J. -B., 1770-1780, Sur le problème de Molyneux, réédité dans F. Markovits (éd.), Diderot, Mérian et l'aveugle, Paris : Flammarion, 1984.

Millikan, R. G., 1993, "Content and vehicle”, dans Eilan, McCarthy \& Brewer (éds), pp. 256-268. 
Morgan, M. J., 1977, Molyneux's Question, Cambridge : Cambridge University Press.

Poincaré, H., 1905, La valeur de la science, Paris : Flammarion.

Poincaré, H., 1907, La science et l'hypothèse, Paris : Flammarion.

Poincaré, H., 1930, Dernières pensées, Paris : Flammarion.

Reid, Th., 1785, Essays on the Intellectual Powers of Man, repris dans Works, W. Hamilton (éd.), vol. I, Edinburgh, 1895.

Rock, I., 1966, The Nature of Perceptual Adaptation, New-Tork : Basic Books.

Roxbee-Cox, J. W., 1970, "Distinguishing the Senses", Mind, 79, pp. 530-550.

Schoemaker, S., 1975, "Functionalism and Qualia", Philosophical Studies, 27, pp. 291-315.Schwartz, R., 1994, Vision, Cambridge, Mass. : Blackwell.

Schoemaker, S., 1982, “The inverted Spectrum”, Journal of Philosophy, 79, 357-381.

Senden, M. von, 1960, Space and Sight, Londres : Methuen

van Gulick, R., 1993, "What difference does consciousness make ?", Philosophical Topics, 17 : 211-230.

White, B.W., Saunders, F.A., Scadden, L., Bach-Y-Rita, P. \& C. C. Collins, 1970, "Seeing with the skin", Perception and Psychophysics, vol. 7, 1, pp. 23-27. 